\section{A rhythmic chest pain}

\author{
Lorenzo Cristoni, Andrea Tampieri, \\ Tiziano Lenzi \\ Emergency Department, Imola Local \\ Health Unit, Imola, Italy
}

\section{Abstract}

A middle-aged man with a history of ischemic heart disease presented at the emergency department having had a forty-minute long precordial pain at home and with an electrocardiogram showing a wide complex tachycardia with left bundle branch block shaped QRS. While preparing for urgent electrical cardioversion, the physician practiced a carotid sinus massage which helped to i) unveil the supraventricular origin of the arrhythmia (by slowing down heart frequency and thus displaying $p$ waves previously hidden) and ii) to exclude that the aberrancy was the expression of a transmural ischemia, thanks to the narrowing of the QRS complex. The final diagnosis was atrial tachycardia. The patient consequently received amiodarone IV and was discharged in normal sinus rhythm. We emphasize the importance of correctly diagnosing any rhythm disorder before administering the treatment, if the patient's clinical condition permits it, in order to ensure the best treatment in urgency and the most appropriate prophylaxis.

\section{Case Report}

A 59-year-old man was admitted to the emergency department (ED) due to precordial pain which started at rest $2 \mathrm{~h}$ before presentation, along with cold sweats, and it had all resolved spontaneously after 40 min since onset.

The 12 leads electrocardiogram (ECG) showed a wide QRS complex tachycardia (Figure 1), with the QRS appearing with a left bundle branch block (LBBB). No previous ECG provided by the patient documented an LBBB. Patient's blood pressure was 140/100, arterial blood oxygen saturation was $98 \%$ and no signs of heart failure were detected on physical examination.

Main laboratory exams at presentation were hemoglobin (Hb) $12.1 \mathrm{~g} / \mathrm{mL}$, glucose 266 $\mathrm{mg} / \mathrm{dL}$, potassium (K) $5 \mathrm{mEq} / \mathrm{L}$, sodium (Na) $140 \mathrm{mEq} / \mathrm{L}$, calcium $(\mathrm{Ca}++) 9.6 \mathrm{mg} / \mathrm{dL}$, creatinine $1.2 \mathrm{mg} / \mathrm{dL}$, and urea $46 \mathrm{mg} / \mathrm{dL}$.

The patient had a history of a long-lasting type 2 mellitus diabetes and hypertension. Also, at 56 he had undergone surgery for coronary artery bypass grafting on the anterior interventricular coronary artery due to an unstable angina.

The patient then had suffered from postoperative atrial fibrillation (AF) and thereafter he had been receiving anticoagulation with warfarin. After sinus rhythm restoration was restored by direct current cardioversion (DCC), warfarin treatment was discontinued and prophylactic treatment with flecainide 50 mg twice daily (bid) was then started.

Home treatment at presentation was metoprolol $50 \mathrm{mg}$ bid, flecainide $50 \mathrm{mg}$ bid, lercanidipine $10 \mathrm{mg}$ once daily (qd), furosemide 25 $\mathrm{mg}$ qd, valsartan $40 \mathrm{mg}$ qd, acetylsalicylic acid $100 \mathrm{mg}$ qd, lansoprazole $30 \mathrm{mg} \mathrm{qd}$, metformine $500 \mathrm{mg}$ three times a day, and glimepiride $2 \mathrm{mg}$ qd. He reported that the current chest pain was similar to that of the angina suffered in the past.

A carotid sinus massage was first performed during continuous ECG monitoring, while preparing for urgent DCC. A transient slowing of atrioventricular conduction revealed the presence of $p$ waves previously hidden in the QRS complex and in the T waves (Figure 2) and resulted in the narrowing of QRS complex.

The initial hypothesis of an acute coronary syndrome (anginal chest pain and alleged new-onset of LBBB in the context of ischemic heart disease) was thus less likely because of the frequency dependence of the LBBB.

This, along with the cessation of ischemia related symptoms, led us not to consider the arrhythmia unstable any longer and thus not to require urgent DCC.

We administered $300 \mathrm{mg}$ amiodarone IV over $30 \mathrm{~min}$, which allowed to confirm the diagnosis of atrial tachycardia (AT) in 12-lead ECG registered at the end of the infusion (Figure 3) thanks to the slowdown of atrioventricular conduction from 1:1 to 2:1 (note in particular the $p$ waves in lead V1). The ventricular rate had in fact reduced to half of that seen at the first ECG, where the conduction was 1:1. Also, the QRS narrowed just like when carotid sinus massage was performed, thereby demonstrating the rate-dependent nature of the LBBB observed during the faster conduction.

Infusion of amiodarone was maintained and the patient received $600 \mathrm{mg}$ over $12 \mathrm{~h}$. Echocardiogram was performed when still in the emergency department and it revealed a marked left ventricular hypertrophy (the interventricular septum measured $21 \mathrm{~mm}$ thickness, suggestive for a diagnosis of nonobstructive hypertrophic cardiomyopathy), an ejection fraction of $45 \%$, and a moderate mitral insufficiency.

Troponin I has reached a pick of $0.071 \mathrm{ng} / \mathrm{mL}$ and the patient was then studied with angiography, which revealed stable coronary disease (subcritical stenosis with functioning graft) compared to the previous study.
Correspondence: Tiziano Lenzi, Emergency Department, Imola Local Health Unit, viale Amendola 2, 40026 Imola, Italy.

Tel. +39.054.2662924 - Fax: +39.054.2662925.

E-mail: t.lenzi@ausl.imola.bo.it

Key words: rhythmic, chest, pain.

Contributions: the authors contributed equally.

Conflict of interests: the authors declare no potential conflict of interests.

Received for publication: 20 February 2013.

Revision received: 26 July 2013.

Accepted for publication: 14 August 2013.

This work is licensed under a Creative Commons Attribution 3.0 License (by-nc 3.0).

() Copyright L. Cristoni et al., 2013

Licensee PAGEPress, Italy

Emergency Care Journal 2013; 9:e25

doi:10.4081/ecj.2013.e25

Angiography was performed without adjunctive provocative test or myocardial nuclear imaging because of the typical anginal pain accompanying the tachyarrhythmia paroxysm, which was then regarded as a positive stress test.

\section{Discussion}

This patient, with a history of ischemic heart disease, had a typical ischemic chest pain and showed a tachycardia with QRS complex with the appearance of an LBBB, not previously documented.

These findings were compatible with an acute coronary syndrome and would prompt urgent treatments aiming at reducing the ventricular rate, and consequently the oxygen consumption of the myocardium, along with the hemodinamyc service alert for urgent percutaneus coronary intervention due to probable transmural ischemia.

Anyway, even a ventricular tachycardia (VT) could be responsible of the anginal pain and, despite QRS complex had the shape of LBBB, we were not to exclude $a$ priori this hypothesis. Besides, the lack of previously documented LBBB may lead to consider, in the differential diagnosis, all regular supraventricular tachycardia (atrio ventricular node reentrant tachycardia, orthodromic atrio ventricular reentrant tachycardia, atrial flutter or atrial tachycardia), with tachycardia-dependet or pre-existing aberrancy. An antidromic atrio ventricular reentrant tachycardia, i.e. with anteograde conduction over an accessory pathway (preexcited) and consequent widening of QRS, could 
also be considered in the differential diagnosis, albeit with a ventricular rate below average. ${ }^{1}$ It is also worth mentioning that the current prophylactic treatment with flecainide, which prolong QRS duration by slowing down intraventricular conduction, could be itself a potential cause of QRS prolongation in all supraventricular tachycardia. ${ }^{2}$

The heart rate showed by the first ECG was $156 /$ min and this could first lead to think about an atrial flutter with 2:1 conduction and LBBB because atrial rate in atrial flutter is typically about 300 beats/min.

In addition, the treatment with flecainide could lead to speculate even the presence of an atrial flutter with 1:1 conduction, attributable to a drug-induced slowdown in the frequency of activation of $\mathrm{F}$ waves, and consequent tachycardia-dependent LBBB.

In addition, it could also be an AT with 1:1 conduction because the heart rate is typically $<250$ beats/min in this type of arrhythmia. However, the presence of LBBB made this hypothesis a little less probable because, in the majority of ATs, if aberrance is present we observe a right bundle branch block (RBBB). ${ }^{3}$ Our case, however, showed a kind of AT with LBBB.

According to the standard emergency approach to wide complex tachycardia recommended by international guidelines, in particular in patients with ischemic heart disease where it is more common to occur, VT is however the diagnostic hypothesis to be taken more into account for the danger of treating a VT as a supraventricular tachycardia (SVT). ${ }^{4}$

Anyway, if we carefully analyze the ECG in Figure 1 we would not identify the classic diagnostic criteria for VT such as atrio-ventricular dissociation, fusion and/or capture complex, concordant precordial pattern and an interval $>100 \mathrm{~ms}$ from the beginning of the QRS to the nadir of $\mathrm{S}$ wave in precordial leads. ${ }^{5}$ Moreover, the ECG in Figure 1 shows QRS complex with typical LBBB, which makes a SVT with aberrancy an even more likely diagnosis. A definitive exclusion of VT can also be achieved by means of the recently proposed algorithm that focuses only on aVR lead because of the absence of each of the four sufficient diagnostic criteria for VT in this lead which are an initial $\mathrm{R}$ wave, width of an initial $\mathrm{r}$ or $\mathrm{q}$ wave $>40$ ms, notching on the initial downstroke of a predominantly negative $\mathrm{QRS}$ complex and ventricular activation-velocity ratio $<1$, i.e. the ratio of the vertical excursion (in millivolts) recorded during the initial and terminal $40 \mathrm{~ms}$ of the QRS complex $<1$. $^{6}$

The application of the aforementioned criteria to differentiate VT from SVT with aberrancy could easily take an expert physician to the right diagnosis of this case just from the first ECG, but emergency physicians do not usually have a specific cardiology curriculum and usu- ally they deal with unstable patients who often require a timely treatment prior to a precise diagnosis. In the absence of a known pre-existing aberrancy it is therefore common in the ED to treat all wide QRS tachycardia as VT, as ACLS guidelines indeed suggest.

Anyway, despite the anxiety induced by patient history and ECG, we considered important to pursue the right diagnosis before the restoration of normal sinus rhythm because of the different prognostic significance of each arrhythmia and because in certain circumstances the treatment may be ineffective or harmful.

Once sinus rhythm is restored, in fact, the problem of the prevention of relapses has to be addressed, and prophylaxis must be tailored according to the malignancy of the arrhythmia.

Prevention of recurrence of VT has prognostic value, and a way of being pursued, which is very different from SVT prophylaxis of episodes of SVT. ${ }^{1,7}$

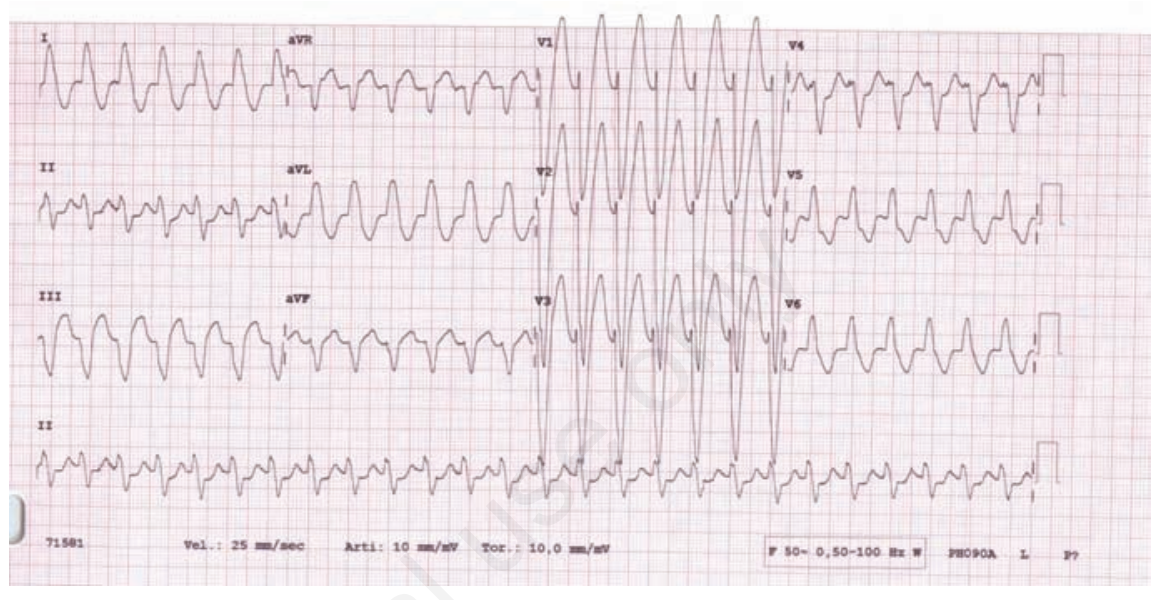

Figure 1. Electrocardiogram showing wide QRS complex tachycardia.

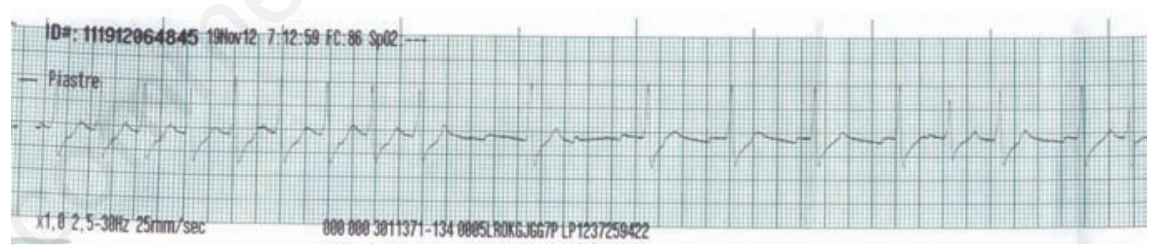

Figure 2. Electrocardiogram showing a transient slowing of atrioventricular conduction.

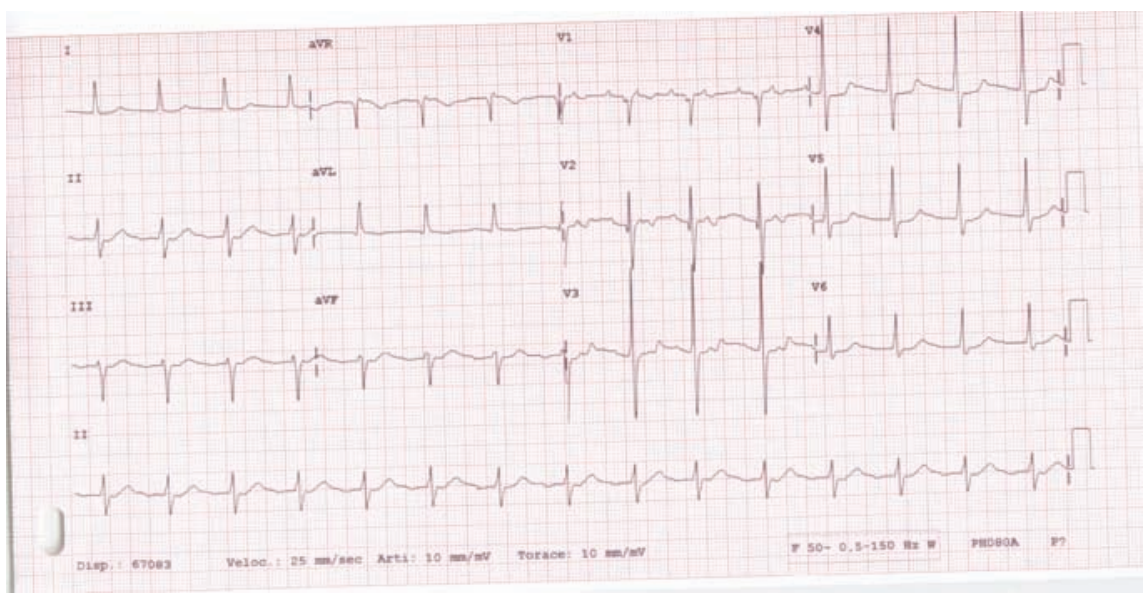

Figure 3. Electrocardiogram registered at the end of the infusion. 
Hence, it follows that the differential diagnosis should be sought as much as possible before administering the treatment when dealing with a wide QRS tachycardia. Vagal stimulation via carotid sinus massage, while preparing for urgent DCC (which is the standard treatment of unstable tachyarrhythmia) ${ }^{4}$ was then the first attempt to understand the kind of arrhythmia we were dealing with. For practical convenience, we preferred this to adenosine administration, as indicated by ACLS guidelines to help in the differential diagnosis of wide complex stable tachycardia.

We were however aware of the existence of a variety of VT, specifically the idiopathic VT, which typically may reveal LBBB as our case, that can be terminated by vagal stimulation, in particular by adenosine infusion. Thus, the eventual conversion to sinus rhythm by carotid sinus massage would not have been a conclusive evidence for the supraventricular origin of the arrhythmia. ${ }^{8,9}$

However, idiopathic VT is more likely to occur in young people without structural heart disease and thus at least a carotid sinus massage was attempted.

The same diagnostic dilemma between VT and SVT with LBBB could obviously have been left unsolved in case sinus rhythm was restored by DCC. However, after not considering the arrhythmia as unstable anymore, the DCC was not considered as the first treatment option.

Anyway due, to the frequent typical origin of AT from increased automaticity of an ectopic focus, DCC would likely have proved ineffective in terminating the arrhythmia. ${ }^{1}$

In this regard, however, we should specify that the surface ECG does not allow us to investigate the electrogenesis of AT so that we can not exclude that the tachycardia mechanism is micro-re-entry or triggered automaticity which respond to DCC. Guidelines in fact recommend DCC as the first line treatment of AT with haemodynamic instability and its use is also recommended in case of resistance to drug treatment. ${ }^{1}$

The infusion of amiodarone, recommended as the first option in stable wide complex tachycardia, ${ }^{4}$ would have nevertheless made it possible to get to the right diagnosis without any previous vagal stimulation, and our case clearly demonstrated it.

ACLS guidelines warns against the use of any kind of antiarrhythmics with inotropic negative effect in case of wide QRS tachycardia and we strictly observed this indication.

Infusion of amiodarone and DCC are the two main options for treatment in this case, and DCC is preferred in case of hemodynamic instability (class I B recommendation). ${ }^{4}$

Anyway, thanks to the rapid diagnosis of AT obtained with carotid sinus massage, instead of administering $150 \mathrm{mg}$ amiodarone IV over 10 min as ACLS guidelines suggest to treat a wide QRS tachycardia without hemodynamic instability, we started the treatment of what we have come to consider a haemodynamic stable supraventricular arrhythmia by administering amiodarone $5 \mathrm{mg} / \mathrm{kg}$ (i.e. $300 \mathrm{mg}$ for our patient) IV over 30 min followed by infusion of amiodarone $600 \mathrm{mg}$ over the next $12 \mathrm{~h}$, with the goal of both lowering ventricular response and restoring sinus rhythm by suppressing the tachycardia focus. ${ }^{10}$

\section{Conclusions}

Coronary artery disease is recognized as a contraindication to the use of class Ic antiarrhythmics. International guidelines are still cautioning doctors from using class Ic antiarrhytmic drugs in patients with structural and ischemic heart disease and the decision to submit the patient to a chronic prophylaxis with flecainide for the prevention of the recurrences of atrial fibrillation, although at subtherapeutic dosage, is therefore questionable.

Moreover the subtherapeutic dosage of flecainide prescribed (50 mg bid), which suggests a compromise choice between the aim of preventing recurrences of $\mathrm{AF}$ and the risk of adverse events, is not supported by evidence of the effectiveness of such low doses of the drug, which can be used only in severe renal insufficiency. Treatment with flecainide was permanently discontinued. Anticoagulation with warfarin has been added to antiplatelet therapy with acetylsalicylic acid.

The choice to start anticoagulation was due more to the previous diagnosis of AF than AT, because there is no clear scientific evidence dictating AT as an indication to anticoagulation. Indeed, a CHA2DS2VASc score of 3 points (hypertension, mellitus diabetes and documented vascular disease) made warfarin treatment actually indicated in a patient with history of AF. Metoprolol has been confirmed at a dose of $50 \mathrm{mg}$ bid and the rest of home therapy remained unchanged. The patient was discharged in normal sinus rhythm after 5 days of hospitalization.

\section{References}

1. Blomström-Lundqvist C, Scheinman MM. ACC/AHA/ESC guidelines for the management of patients with supraventricular arrhythmias - executive summary. J Am Coll Cardiol 2003;42:1493-531.

2. Oreto G, Luzza F, Satullo G, et al. [Tachicardia a complessi larghi]. [Article in Italian]. Available from: http://www. giornaled icardiologia.it/allegati/00580_2009_09/fulltext/200909_04\%20580-595.pdf

3. Chou TC, Knilans TK. Electrocardiography in clinical practice adult and pediatric. 4th ed. Philadelphia, PA: Saunder; 2002.

4. Travers AH, Rea TD, Bobrow BJ, et al. 2010 American Heart Association guidelines for cardiopulmonary resuscitation and emergency cardiovascular care science. Circulation 2010;122:S729-67.

5. Brugada P, Brugada J, Mont L. A new approach to the differential diagnosis of a regular tachycardia with a wide $\mathrm{QRS}$ complex. Circulation 1991;83:1649-59.

6. Vereckei A, Duray G, Szénási G, et al. New algorithm using only lead aVR for differential diagnosis of wide $\mathrm{QRS}$ complex tachycardia. Heart Rhythm 2008;5:89-98.

7. European Heart Rhythm Association. ACC/AHA/ESC 2006 guidelines for management of patients with ventricular arrhythmias and the prevention of sudden cardiac death: a report of the American College of Cardiology/American Heart Association Task Force and the European Society of Cardiology Committee for Practice Guidelines (Writing Committee to Develop guidelines for management of patients with ventricular arrhythmias and the prevention of sudden cardiac death). $\mathrm{J}$ Am Coll Cardiol 2006;48:e247-346.

8. Wilber DJ, Baerman J, Olshansky B, et al. Adenosine-sensitive ventricular tachycardia. Clinical characteristics and response to catheter ablation. Circulation 1993;87: 126-34

9. Marill KA, Wolfram S, Desouza IS, et al. Adenosine for wide-complex tachycardia: efficacy and safety. Crit Care Med 2009;37: 2512-8.

10. Camm J, Kirchhof P, Lip GYH, et al. Guidelines for the management of atrial fibrillation. The task force for the management of atrial fibrillation of the European society of cardiology (ESC). Eur Heart J 2010;31:2369-429. 